\title{
On a Class of Dual Risk Model with Dependence based on the FGM Copula
}

\author{
Hua Dong Zaiming Liu \\ School of Mathematical Sciences \\ Central South University \\ Changsha, China \\ Email: sddh1978@126.com
}

\begin{abstract}
In this paper, we consider an extension to a dual model under a barrier strategy, in which the innovation sizes depend on the innovation time via the FGM copula. We first derive a renewal equation for the expected total discounted dividends until ruin. Some differential equations and closed-form expressions are given for exponential innovation sizes. Then the optimal dividend barrier and the Laplace transform of the time to ruin are considered. Finally, a numerical example is given..
\end{abstract}

Index Terms—Dividends, dependence, barrier strategies.

\section{INTRODUCTION}

It is well known that the classical risk process has been studied profoundly, e.g. Asmussen[1], Gerber and Shiu [2, 3], Dufresne and Gerber [4]. For the classical risk model, it is assumed that the inter-claim times between two successive claims and the claim amounts are independent. Such an assumption may be inappropriate in real world. To avoid the restriction, some risk models with dependence structure between inter-claim times and claim sizes are proposed. Among them, Albrecher and Boxma[5] have proposed an extension to the classical compound Poisson risk model in which the distribution of the time between two successive claims depends on the the previous claim size. Albrecher and Teugels [6] considered an risk model with arbitrary dependence structure between the inter-claim times and the subsequent claim size through a copula. In Boudreault et al.[7], they assumed that the claim size depend on the inter-claim times.

The barrier strategy was first studied in De Finetti [8]. Barrier strategies for the compound Poisson process risk model have been studied in detail by numerous authors, e.g. Dickson and Waters[9], Landriault[10], Lin et al.[11] and Lin and Pavlova[12].

Recently, there has been growing interest in a model which is dual to the classical risk model. See Asmussen [1], Albrecher et al. [13], Avanzi et al. [14], Avanzi and Gerber [15], Ng [16], Song et al. [17] and references therein. Avanzi et al. [14] considered the expected total discounted dividends until ruin for the dual model under the barrier strategy by means of integro-differential equation.

Avanzi and Gerber [15] extended some results of Avanzi et al. [14] to a dual model perturbed by diffusion. Ng [16] generalized the study of Avanzi et al. [14] to a dual model with dividend threshold strategy. Here, we aim at extending some results of Avanzi et al. [14] to a dual risk model with dependence structure which is based on the Farlie-Gumbel-Morgenstern (FGM)(see Nelsen [18]) copula.

Consider the dual model

$U(t)=u-c t+\sum_{i=1}^{N(t)} X_{i}=u-c t+S(t), \quad t \geq 0$,

where $u \geq 0$ is the initial surplus, $c>0$ is the constant rate of expenses and $S(t)$ represents the aggregate gains up to time $t$. The innovation number process $\{N(t), t \geq 0\}$ is a renewal process with inter-innovation times $\left\{W_{j}\right\}_{j \geq 1}$, where $\left\{W_{j}\right\}_{j \geq 1}$ is a sequence of strictly positive and independent random variables (r.v.) with probability density function (p.d.f.) $f_{W}$ and distribution function (d.f.) $F_{W}$. Throughout this paper, it is assumed that $W$ has an exponential distribution with mean $1 / \lambda$. The innovation size (gains) r.v.'s $\left\{X_{j}\right\}_{j \geq 1}$, where $X_{j}$ corresponds to the amount of the $j$ th innovation, are assumed to be a sequence of strictly positive and independent r.v.'s with a common p.d.f. $f_{X}$, d.f. $F_{X}$, mean $\mu_{X}$ and Laplace transform $\hat{f}_{X}$. We assume that $\left\{\left(X_{j}, W_{j}\right)\right\}$ forms a sequence of i.i.d. random vectors with joint p.d.f. $f_{X, W}(x, t)$ for $t \geq 0$ and $x \geq 0$. It is clear that the increments $\left\{X_{j}-c W_{j}, j=1,2, \cdots\right\}$ of the surplus process are still independent. To ensure that ruin will not occur almost surely, we assume that

$\mu=\lambda E\left(X_{i}-c W_{i}\right)=\lambda \mu_{X}-c>0, i=1,2, \cdots$.

Furthermore, we assume that the joint distribution of $(X, W)$ is defined with the FGM copula (see Nelsen [18]), which is defined by

$C(u, v)=u v+\theta u(1-u) v(1-v),-1 \leq \theta \leq 1$.

Given (2), the joint distribution function of $F_{X, W}(x, t)$ is defined by

$$
\begin{aligned}
& F_{X, W}(x, t)=C\left(F_{X}(x), F_{W}(t)\right) \\
& \quad=F_{X}(x) F_{W}(t)\left(1+\theta\left(1-F_{X}(x)\right)\left(1-F_{W}(t)\right)\right) .
\end{aligned}
$$

Thus, the joint p.d.f. is given by 


$$
\begin{aligned}
& f_{X, W}(x, t)=c\left(F_{X}(x), F_{W}(t)\right) f_{X}(x) f_{W}(t) \\
& \quad=f_{X}(x) f_{W}(t)\left(1+\theta\left(1-2 F_{X}(x)\right)\left(1-2 F_{W}(t)\right)\right) .
\end{aligned}
$$

In this paper, we assume that the dividends are paid according to a barrier strategy, say with the parameter $b>0$. Whenever the surplus exceeds the barrier $\mathrm{b}$, the excess is paid out immediately as a dividend. Let $U_{b}(t)$ be the modified surplus process with initial surplus $U_{b}(0)=u$ under the above barrier strategy $b$.

Let $V(u ; b)=E\left[\int_{0}^{T} e^{-\delta t} \mathrm{dD}(\mathrm{t})\right]$ denote the expectation of the discounted dividends until ruin with the boundary condition $V(0 ; b)=0$, where $T=\inf \left\{t \geq 0: U_{b}(t)=0\right\}(T=\infty$ if ruin does not occur) is the time of ruin and $\delta>0$ is the force of interest to discount the dividends.

Noticing that

$$
V(u ; b)=u-b+V(b ; b), \quad u>b,
$$

we will mainly discuss the model for $0 \leq u \leq b$.

The paper is organized as follows. In Section 2, we start by deriving an integro - differential equation satisfied by $V(u ; b)$. Then the integro - differential equation leads to some differential equations and closed form expressions for exponential gains. In Section 3, we discuss how we can use the method of Laplace transforms to obtain the expected total discounted dividends. A renewal equation is also given. In section 4, the optimal dividend barrier is considered. The Laplace transform of differential equation leads to some differential equations and closed form expressions for exponential gains. In Section 3, we discuss how we can use the method of Laplace transforms to obtain the expected total discounted dividends. A renewal equation is also given. In section 4, the optimal dividend barrier is considered. The Laplace transform of the time to ruin is studied in Section 5 for exponential gains. Finally, a numerical illustration is given in Section 6

\section{EXPRESSIONS FOR $V(u ; b)$}

Theorem 2.1 For $0<u<b, V(u ; b)$ satisfies the following integro - differential equation

$$
\begin{array}{r}
\delta V(u ; b)+c V^{\prime}(u ; b)+V(u ; b) \int_{0}^{\infty} f_{X, W}(x, 0) \mathrm{d} x \\
-\int_{0}^{\infty} V(u+x ; b) f_{X, W}(x, 0) \mathrm{d} x=0,
\end{array}
$$

where

$f_{X, W}(x, 0)=\lambda f_{X}(x)+\theta \lambda\left(1-2 F_{X}(x)\right) f_{X}(x)$.

Proof. Choosing a time $\tau$ small enough such that $u-c \tau>0$, one obtains

$$
\begin{gathered}
V(u ; b)=e^{-\delta \tau} V(u-c \tau ; b) \int_{\tau}^{\infty} \int_{0}^{\infty} f_{X, W}(x, t) \mathrm{d} x \mathrm{~d} t \\
+\int_{0}^{\tau} \int_{0}^{\infty} e^{-\delta t} V(u-c t+x ; b) f_{X, W}(x, t) \mathrm{d} x \mathrm{~d} t .
\end{gathered}
$$

Differentiating the above equation with respect to $\tau$ and then letting $\tau=0$ yield (4).

Corollary 2.1 For $0<u<b$, if the gains are exponentially distributed with mean $1 / \beta$, then $V(u ; b)$ satisfies the following integro-differential equation

$$
\begin{aligned}
& c V^{\prime}(u ; b)+(\lambda+\delta-2 \lambda \theta) V(u ; b) \\
& -\lambda\left(1-\theta-\theta e^{-\beta(b-u)}\right) e^{-\beta(b-u)} V(b ; b) \\
& -\lambda \beta \int_{u}^{b}\left(1-\theta-2 \theta e^{-\beta(y-u)}\right) e^{-\beta(y-u)} V(y ; b) d y \\
& -\frac{\lambda}{2 \beta}\left(2(1-\theta)+\theta e^{-\beta(b-u)}\right) e^{-\beta(b-u)}=0 .
\end{aligned}
$$

Corollary 2.2 If the profits are exponentially distributed with p.d.f. $f_{X}(x)=\beta e^{-\beta x}, x \geq 0$ and $\theta \in[-1,0) \cup(0,1)$, then $V(u ; b)$ satisfies differential equation

$$
\begin{aligned}
& c V^{\prime \prime \prime}(u ; b)+(\lambda+\delta-3 c \beta) V^{\prime \prime}(u ; b)+2 \delta \beta^{2} V(u ; b) \\
& -\beta(2 \lambda+3 \delta-2 c \beta-\lambda \theta) V^{\prime}(u ; b)=0,
\end{aligned}
$$

for $0<u<b$.

Proof. Applying the operator $\left(\frac{d}{d t}-\beta\right)\left(\frac{d}{d t}-2 \beta\right)$ to (7), then we obtain (8).

From (8) and the boundary condition $V(0 ; b)=0$, we have

$$
\begin{aligned}
V(u ; b) & =\sum_{k=0}^{2} C_{k} e^{r_{k} u}, \\
0 & \leq u \leq b, \theta \in[-1,0) \cup(0,1),
\end{aligned}
$$

where $r_{k}(k=1,2,3)$ are the solutions of the equation

$$
c s+\lambda+\delta=\frac{\lambda \beta(1-\theta)}{\beta-s}+\frac{2 \lambda \theta \beta}{2 \beta-s} .
$$

To determine $C_{k}=C_{k}(b)$, we insert (9) into (7) and obtain

$$
\begin{aligned}
& \sum_{k=0}^{2} C_{k}\left(c r_{k}+\lambda+\delta-2 \lambda \theta\right) e^{r_{k} u} \\
& -\lambda\left[1-\theta-\theta e^{-\beta(b-u)}\right] \sum_{k=0}^{2} C_{k} e^{r_{k} b-\beta(b-u)} \\
& -\lambda \beta \sum_{k=0}^{2} C_{k} \int_{u}^{b}\left[1-\theta-2 \theta e^{-\beta(y-u)}\right] e^{-\beta(y-u)} e^{r_{k} y} d y \\
& -\frac{\lambda}{2 \beta}\left[2(1-\theta)+\theta e^{-\beta(b-u)}\right] e^{-\beta(b-u)}=0 .
\end{aligned}
$$

By matching the coefficients in (11) one obtains 
$\sum_{k=0}^{2} \frac{C_{k} r_{k}}{\beta-r_{k}} e^{r_{k} b}-\theta \sum_{k=0}^{2} \frac{C_{k} r_{k}}{\beta-r_{k}} e^{r_{k} b}=\frac{1-\theta}{\beta}$,

which is the coefficient of $\lambda e^{-\beta(b-u)}$, and

$$
\theta \sum_{k=0}^{2} \frac{C_{k} r_{k}}{2 \beta-r_{k}} e^{r_{k} b}=-\frac{\theta}{2 \beta},
$$

which is the coefficient of $\lambda e^{-2 \beta(b-u)}$. Combining with the boundary condition $V(0 ; b)=0$, we have

$$
\sum_{k=0}^{2} C_{k}=0
$$

Define $M$ as the coefficient matrix of the system (12) (14) and the column vector $\eta=(\beta,-1 / 2 \beta, 0)^{\top}$. Let $M_{i}$ be the matrix obtained from $n M$ by replacing its $i$ th column by $\eta$ for $\mathrm{i}=1,2,3$. Denote the determinant of a matrix by $\operatorname{det}(\cdot)$. Then, we have $C_{i}=(\operatorname{det}(M))^{-1} \operatorname{det}\left(M_{i+1}\right), i=0,1,2$, provided that $\operatorname{det}(M) \neq 0$. Some careful calculations give

$$
\begin{aligned}
\operatorname{det}(M)= & {\left[\frac{1}{\left(\beta-r_{1}\right)\left(2 \beta-r_{2}\right)}-\frac{1}{\left(\beta-r_{2}\right)\left(2 \beta-r_{1}\right)}\right] } \\
& \times r_{1} r_{2} e^{\left(r_{1}+r_{2}\right) b} \\
& +\left[\frac{1}{\left(\beta-r_{2}\right)\left(2 \beta-r_{0}\right)}-\frac{1}{\left(\beta-r_{0}\right)\left(2 \beta-r_{2}\right)}\right] \\
& \times r_{0} r_{2} e^{\left(r_{0}+r_{2}\right) b} \\
& +\left[\frac{1}{\left(\beta-r_{0}\right)\left(2 \beta-r_{1}\right)}-\frac{1}{\left(\beta-r_{1}\right)\left(2 \beta-r_{0}\right)}\right] \\
& \times r_{1} r_{0} e^{\left(r_{1}+r_{0}\right) b} \\
\operatorname{det}\left(M_{1}\right)= & \left(\frac{1}{\beta\left(2 \beta-r_{2}\right)}+\frac{1}{2 \beta\left(\beta-r_{2}\right)}\right) r_{2} e^{r_{2} b} \\
& -\left(\frac{1}{\beta\left(2 \beta-r_{0}\right)}+\frac{1}{2 \beta\left(\beta-r_{0}\right)}\right) r_{0} e^{r_{0} b}, \\
\operatorname{det}\left(M_{2}\right)= & \left(\frac{1}{\beta\left(2 \beta-r_{0}\right)}+\frac{1}{2 \beta\left(\beta-r_{0}\right)}\right) r_{0} e^{r_{0} b} \\
& -\left(\frac{1}{\beta\left(2 \beta-r_{1}\right)}+\frac{1}{2 \beta\left(\beta-r_{1}\right)}\right) r_{1} e^{r_{1} b}, \\
\operatorname{det}\left(M_{3}\right)= & \left(\frac{1}{\beta\left(2 \beta-r_{1}\right)}+\frac{1}{2 \beta\left(\beta-r_{1}\right)}\right) r_{1} e^{r_{1} b} \\
& -\left(\frac{1}{\beta\left(2 \beta-r_{2}\right)}+\frac{1}{2 \beta\left(\beta-r_{2}\right)}\right) r_{2} e^{r_{2} b} .
\end{aligned}
$$

Remark 2.1 When $\theta \in(0,1)$, one can show that

$$
-\frac{\lambda+\delta}{c}<r_{0}<0<r_{1}<\beta<r_{2}<2 \beta ;
$$

When $\theta \in[-1,0)$, one can show that

$$
-\frac{\lambda+\delta}{c}<r_{0}<0<r_{1}<\beta<2 \beta<r_{2} \text {. }
$$

In a word, (10) has one negative root and two positive roots.

Corollary 2.3 If the profits are exponentially distributed with p.d.f. $f_{X}(x)=\beta e^{-\beta x}, x \geq 0$, and $\theta=1$, then $V(u ; b)$ satisfies differential equation $c V^{\prime \prime}(u ; b)+(\lambda+\delta-2 \beta c) V^{\prime}(u ; b)-2 \beta \delta V(u ; b)=0$,

for $0<u<b$.

Proof. Setting $\theta=1$ in (10), we have

$$
\begin{array}{r}
c V^{\prime}(u ; b)-(\lambda-\delta) V(u ; b)+\lambda e^{-2 \beta(b-u)} V(b ; b) \\
+2 \lambda \beta \int_{u}^{b} e^{-2 \beta(y-u)} V(y ; b) d y-\frac{\lambda}{2 \beta} e^{-2 \beta(b-u)}=0 .
\end{array}
$$

By applying the operator $\left(\frac{d}{d u}-2 \beta\right)$ to this equation, we obtain (15). From the boundary condition $V(0 ; b)=0$ and (15), it follows that

$$
\begin{array}{r}
V(u ; b)=\frac{\lambda}{2 \beta} \frac{e^{r_{1} u}-e^{r_{0} u}}{\left(c r_{1}+\delta\right) e^{r_{1} b}-\left(c r_{0}+\delta\right) e^{r_{0} b}}, \\
0 \leq u \leq b,
\end{array}
$$

where $r_{0}<0<r_{1}$ are solutions of equation

$$
c x^{2}+(\lambda+\delta-2 \beta c) x-2 \beta \delta=0 .
$$

Corollary 2.4 If the profits are exponentially distributed with p.d.f. $f_{X}(x)=\beta e^{-\beta x}, x \geq 0$, and $\theta=0$, then for $0<u<b$

$c V^{\prime \prime}(u ; b)+(\lambda+\delta-\beta c) V^{\prime}(u ; b)-\beta \delta V(u ; b)=0$,

which is (3.2) in Avanzi et al. [14]. Thus

$V(u ; b)=\frac{\lambda}{\beta} \frac{e^{r_{1} u}-e^{r_{0} u}}{\left(c r_{1}+\delta\right) e^{r_{1} b}-\left(c r_{0}+\delta\right) e^{r_{0} b}}, \quad 0 \leq u \leq b$,

where $r_{0}<0<r_{1}$ are solutions of equation

$$
c x^{2}+(\lambda+\delta-\beta c) x-\beta \delta=0 .
$$

Let $T_{u, b}=\inf \{t \geq 0, U(t) \geq b, 0<t<T\}$ be the time of the first upcrossing of the surplus process through $b$ from $u$ without ruin occurring. Then

$$
V(u ; b)=E\left[e^{-\delta T_{u, b}}\right] V(b ; b),
$$

which means that

$$
E\left[e^{-\delta T_{u, b}}\right]=V(u ; b) V(b ; b)^{-1} .
$$

Corollary 2.5 If the profits are exponentially distributed with p.d.f. $f_{X}(x)=\beta e^{-\beta x}, x \geq 0$, then for $\theta=1$

$$
E\left[e^{-\delta T_{u, b}}\right]=\frac{e^{r_{1} u}-e^{r_{0} u}}{e^{r_{1} b}-e^{r_{0} b}},
$$

where $r_{0}, r_{1}$ are solutions of character equation (17); for $\theta=0$, the expression for $E\left[e^{-\delta T_{u, b}}\right]$ is the same as that for $\theta=1$ in the style, but $r_{0}, r_{1}$ are solutions of character equation (18). For $\theta \in(0,1) \cup[-1,0)$,

$$
E\left[e^{-\delta T_{u, b}}\right]=\frac{\sum_{k=0}^{2} C_{k} e^{r_{k} u}}{\sum_{k=0}^{2} C_{k} e^{r_{k} b}} .
$$




\section{RENEWAL EQUATION}

For notational convenience, we denote the Laplace transform of a function $\varsigma$ by $\hat{\varsigma}(s)=\int_{0}^{\infty} e^{-s x} \varsigma(x) \mathrm{d} x$ for $\boldsymbol{R e}(s) \geq 0$, and $\bar{F}_{X}(x)=1-F_{X}(x)$.

In order to get the Laplace transform of $V(u ; b)$, we replace the random variable $u$ by $z=b-u$ as in Avanzi et al. [14]. Denote

$$
W(z ; b)=V(b-z ; b), \quad \text { for } \quad 0 \leq z \leq b .
$$

Then it follows from (7) that

$c W^{\prime}(z ; b)+\lambda W(0 ; b) \bar{F}_{X}(z)\left(1-\theta F_{X}(z)\right)$

$+\lambda \int_{0}^{z} W(x ; b)\left(1+\theta-2 \theta F_{X}(z-x)\right) f_{X}(z-x) \mathrm{d} x$

$-(\lambda+\delta) W(z ; b)+\lambda \int_{z}^{\infty}\left(1-\theta \bar{F}_{X}(x)\right) \bar{F}_{X}(x) \mathrm{d} x=0$,

with boundary condition

$$
\begin{gathered}
W(0 ; b)=V(b ; b), \\
W(b ; b)=0 .
\end{gathered}
$$

We extend the definition of $W(z ; b)$ by (20) to $z \geq 0$ and denote the resulting function by $\omega(z)$. Then

$$
\begin{aligned}
& c \omega^{\prime}(z)-(\lambda+\delta) \omega(z)+\lambda \omega(0) \bar{F}_{X}(z)\left(1-\theta F_{X}(z)\right) \\
& \quad+\lambda \int_{0}^{z} \omega(x)\left(1-\theta+2 \theta \bar{F}_{X}(z-x)\right) f_{X}(z-x) \mathrm{d} x \\
& \left.\quad+\lambda \int_{z}^{\infty}\left(1-\theta \bar{F}_{X}(x)\right) \bar{F}_{X}(x)\right) \mathrm{d} x=0 .
\end{aligned}
$$

with $\omega(0)=V(b ; b)$ and $\omega(b)=0$. Taking Laplace transform on both sizes of (22) and rearranging it, one obtains

$\hat{\omega}(s)=\frac{c \omega(0)+\frac{\lambda \omega(0)\left(\hat{f}_{X}(s)-1\right)+\lambda \theta \Delta}{s}-\lambda \Xi(s)}{c s-(\lambda+\delta)+\lambda(1+\theta) \hat{f}_{X}(s)-\lambda \theta \hat{g}(s)}$,

where

$\Delta=\int_{0}^{\infty} F_{X}(x)\left(1-F_{X}(x)\right) \mathrm{d} x$,

$g(x)=2 F_{X}(x) f_{X}(x)$,

$\Xi(s)=\frac{\hat{f}_{X}(s)-1+s \mu_{X}-\theta(1-s \omega(0))\left(\hat{g}(s)-\hat{f}_{X}(s)\right)}{s^{2}}$.

Let

$\theta_{0}=\frac{\lambda \int_{0}^{\infty} e^{-s x} x^{2} f_{X}(x) \mathrm{d} x}{\lambda \int_{0}^{\infty} e^{-s x} x^{2}\left(g(x)-f_{X}(x)\right) \mathrm{d} x}$

$A(s)=c s-(\lambda+\delta)+\lambda(1+\theta) \hat{f}_{X}(s)-\lambda \theta \hat{g}(s)$, where $A(s)$ is the denominator in (23). It is obvious $A(0)<0, A(\infty)=\lim _{s \rightarrow \infty} A(s)=\infty$.

When $\theta \in\left[-1, \theta_{0}\right)$, the second derivative of $A(s)$ is positive, otherwise the second derivative of $A(s)$ is negative.
Under any circumstance, $A(s)$ has a unique positive zero, which is defined as $\rho$. $\rho$ must be the zero of the numerator of (23). Some careful calculations lead to

$$
\omega(0)=\frac{1}{\rho}+\frac{\mu-\lambda \theta \Delta}{\delta} \text {. }
$$

Remark 3.1 When the gains are exponentially distributed with mean $1 / \beta$, we have $\hat{f}(s)=\frac{\beta}{\beta+s}, \hat{g}(s)=\frac{2 \beta}{\beta+s}-\frac{2 \beta}{2 \beta+s}$. Replacing $s$ with $-s$ in $A(s)$, we know that $A(s)$ is the negative root of the expression on the left - hand side of (10). In this case, $\rho=-r_{0}$.

Remark 3.2 When $\theta=0$, (23) and (24) can be simplified to (7.3) and (7.8) in Avzani et al. [14], respectively.

For any integral real function $\zeta$, denote $T_{r} \zeta(x)=\int_{x}^{\infty} e^{-r(y-x)} \zeta(y) \mathrm{d} y, r \in \mathcal{C}$. (see Dickson and Hipp [19]).

Inverting the Laplace transform in (23) lead to the following Theorem:

Theorem $3.1 \omega(u)$ satisfies the following renewal equation

$\omega(u)=\int_{0}^{u} \omega(u-x) h(x) \mathrm{d} x+K(u), \quad 0<u<b$,

with boundary condition

$$
\omega(b)=0 \text {, }
$$

$$
\omega(0)=\frac{1}{\rho}+\frac{\mu-\lambda \theta \Delta}{\delta},
$$

where

$$
\begin{aligned}
& h(u)=\lambda(1+\theta) T_{\rho} f_{X}(u) / c-\lambda \theta T_{\rho} g(u) / c, \\
& K(u)=\frac{\lambda}{c \rho}\left(\omega(0)-\theta \Delta+\mu_{X}+\theta u-\frac{1}{\rho}\right) \\
& +\frac{\lambda(1+\theta)}{c} \int_{0}^{u}\left(\frac{1}{\rho} T_{\rho} f_{X}(u-x)-\bar{F}_{X}(u-x)\right) \mathrm{d} x \\
& -\omega(0) \int_{0}^{u}\left(\frac{\lambda(1+\theta)}{c} f_{X}(x)-\frac{\lambda \theta}{c \rho} g(x)\right) \mathrm{d} x \\
& +\frac{\lambda \theta}{c \rho} \int_{0}^{u}\left(\int_{u-x}^{\infty} g(y) \mathrm{d} y-\frac{1}{\rho} T_{\rho} g(u-y)\right) \mathrm{d} y \\
& +\frac{\lambda(1+\rho \omega(0))}{c \rho}\left(\frac{\theta}{\rho} T_{\rho} g(u)-\frac{1+\theta}{\rho} T_{\rho} f_{X}(u)\right) .
\end{aligned}
$$

Proof. Noticing that

$$
\begin{aligned}
& \int_{0}^{\infty} e^{-s u} F_{X}(u) \mathrm{d} u=\hat{f}_{X}(s) / s \\
& \int_{0}^{\infty} e^{-s u} \int_{0}^{u} \bar{F}_{X}(u-x) \mathrm{d} x \mathrm{~d} u=\frac{1-\hat{f}_{X}(s)}{s^{2}} .
\end{aligned}
$$

Inverting (23) leads to the renewal (25).

Since $\rho$ is the zero of $A(s)=0$, it must also be the zero of the numerator in (23). Substituting $\rho$ into the 
numerator of (23, noticing $A(\rho)=0$ and some careful calculation lead to (27). (26) is obvious since (21).

Remark 3.3 For (25) to be a defective renewal equation, it remains to show that $\hat{h}(0)<1$. By the property of operator $T_{r}$, we have

$$
\begin{aligned}
\hat{h}(0)= & \frac{\lambda(1+\theta)}{c} T_{0} T_{\rho} f_{X}(0)-\frac{\lambda \theta}{c} T_{0} T_{\rho} g(0) \\
= & \frac{\lambda(1+\theta) \rho}{c}\left(1-\hat{f}_{X}(\rho)\right)-\frac{\lambda \theta \rho}{c}(1-\hat{g}(\rho)) \\
= & \frac{\lambda}{c \rho}-\frac{\lambda(1+\theta)}{c \rho} \hat{f}_{X}(\rho)+\frac{\lambda \theta}{c \rho} \hat{g}(\rho) \\
= & \frac{\lambda}{c \rho}+\frac{\lambda \theta}{c} \int_{0}^{\infty} e^{-\rho x} F_{X}(x) \bar{F}_{X}(x) \mathrm{d} x \\
& -\frac{\lambda}{c} \int_{0}^{\infty} e^{-\rho x} F_{X}(x) \mathrm{d} x,
\end{aligned}
$$

where we have used

$$
\int_{0}^{\infty} e^{-\rho x} F_{X}^{2}(x) \mathrm{d} x=\hat{g}(\rho) / \rho .
$$

For $-1 \leq \theta \leq 0$, it follows from (28) that

$$
\begin{aligned}
\hat{h}(0) & \leq \frac{\lambda}{c \rho}-\frac{\lambda}{c} \int_{0}^{\infty} e^{-\rho x} F_{X}(x) \mathrm{d} x \\
& <\frac{\lambda}{c} \int_{0}^{\infty} \bar{F}_{X}(x) \mathrm{d} x<1 .
\end{aligned}
$$

The last equality is due to the positive security condition (1).

For $0 \leq \theta \leq 1$, we have

$$
\hat{h}(0) \leq \frac{\lambda}{c} \int_{0}^{\infty} e^{-\rho x}\left(1-F_{X}^{2}(x)\right) \mathrm{d} x .
$$

When $\int_{0}^{\infty} e^{-\rho x}\left(1-F_{X}^{2}(x)\right) \mathrm{d} x<\mu_{X}$, (25) is also a defective renewal equation.

\section{THE OPTIMAL DIVIDEND BARRIER}

In this section, we adopt a similar approach to those used in Avanzi et al.[5] and Avanzi et al.[6] to consider the problem of the determination of the optimal barrier. Let $b^{*}$ be the optimal value of $\mathrm{b}$, then $V(u ; b)$ is maximal at $b^{*}$ for each $u$. Thus

$$
\left.\frac{\partial \mathrm{V}(u ; b)}{\partial b}\right|_{b=b^{*}} \equiv 0,
$$

and hence that

$$
\left.\frac{\mathrm{d} V(b ; b)}{\mathrm{d} b}\right|_{b=b^{*}}=V^{\prime}\left(b^{*}-, b^{*}\right),
$$

where $V^{\prime}(b-; b)$ denotes the left derivative of $V(u ; b)$ at $u=b$. Using (3), we can get

$$
V^{\prime}\left(b^{*}-; b^{*}\right)=1=V^{\prime}\left(b^{*}+; b^{*}\right) \text {. }
$$

This phenomenon is the high contact condition in finance literature and the smooth pasting condition in literature on optional stopping problem.

Setting $u=b=b^{*}$ in (4) and using (30), we obtain

$$
V\left(b^{*} ; b^{*}\right)=\frac{\mu}{\delta}-\frac{\lambda \theta}{2 \beta \delta} .
$$

According to Avanzi and Gerber[6], we can set $\omega(0)=\frac{\mu}{\delta}-\frac{\lambda \theta}{2 \beta \delta}$ and obtain the function $\omega(z)$ by inversion of its Laplace transform. Then $b^{*}$ is the zero of $\omega(z)$, and $V\left(u ; b^{*}\right)=\omega\left(b^{*}-u\right), 0 \leq u \leq b^{*}$.

Remark 4.1 When the gains distribution is exponential distribution with mean $1 / \beta$ and $\theta \in[-1,0) \cap(0,1)$, we can easily get the optimal dividend barrier $b^{*}$ as Avanzi and Gerber[6] did. It follows from (30) and (9) that

$$
C_{k}^{\prime}\left(b^{*}\right)=0, \quad k=0,1,2 .
$$

Now differentiating (12) and (13) with respect to $b$, setting $b=b^{*}$ and applying (33), we get

$$
\begin{aligned}
& \sum_{k=0}^{2} \frac{C_{k}\left(b^{*}\right) r_{k}^{2}}{\beta-r_{k}} e^{r_{k} b^{*}}=0, \\
& \sum_{k=0}^{2} \frac{C_{k}\left(b^{*}\right) r_{k}^{2}}{2 \beta-r_{k}} e^{r_{k} b^{*}}=0 .
\end{aligned}
$$

Then solving the linear system for $C_{0}\left(b^{*}\right), C_{1}\left(b^{*}\right), C_{2}\left(b^{*}\right)$ composed by (14), (34) and (35), we can get the $b^{*}$ which satisfies $\omega\left(b^{*}\right)=0$.

Remark 4.2 When the gains distribution is exponential distribution with mean $1 / \beta$ and $\theta=1$, there is a closed form expressions for $b^{*}$. From (16) we obtain

$$
b^{*}=\frac{1}{r_{1}-r_{0}} \ln \frac{r_{0}\left(c r_{0}+\delta\right)}{r_{1}\left(c r_{1}+\delta\right)},
$$

where $r_{0}$ and $r_{1}$ are the solutions of (17) with $r_{0}<0<r_{1}$.

\section{LAPLACE TRANSFORM OF THE TIME TO RUIN}

In this section, we consider the Laplace transform of the time of ruin $\mathrm{T}$ for the surplus process $U_{b}(t)$ under the barrier strategy. Let

$$
\Psi_{\delta}(u)=E\left[e^{-\delta T} \mid U_{b}(0)=u\right], \quad u>0,
$$

be the expected present value 1 due at the time of ruin. As a function of $\delta$, it is the Laplace transform of $T$. As a function of $u$, it is easy to see that $\Psi_{\delta}(u)=1$ for $u \leq 0$.

Noting that $\Psi_{\delta}(u)=\Psi_{\delta}(b)(u>b)$, we only discuss $\Psi_{\delta}(u)$ for $0<u \leq b$.

Theorem 5.1. Suppose that the profits are exponentially distributed with p.d.f. $f_{X}(x)=\beta e^{-\beta x}, x \geq 0$, then $\Psi_{\delta}(u)$ satisfies the following integro - differential equation

$$
\begin{aligned}
& c \Psi_{\delta}^{\prime}(u)+(\lambda+\delta) \Psi_{\delta}(u)-\lambda(1+\theta) \Psi_{\delta}(b) \bar{F}(b-u) \\
& +\lambda \theta \Psi_{\delta}(u)\left(1-F^{2}(b-u)\right) \\
& -\lambda(1+\theta) \int_{0}^{b-u} \Psi_{\delta}(u+x) f(x) \mathrm{d} x \\
& +\lambda \theta \int_{0}^{b-u} \Psi_{\delta}(u+x) g(x) \mathrm{d} x=0, \\
& \text { for } 0 \leq u \leq b .
\end{aligned}
$$

Proof. The proof is similar to theorem 2.1.

Corollary $\mathbf{5 . 1}$ When the gains distribution is exponential distribution with mean $1 / \beta$ and 
$\theta \in[-1,0) \cap(0,1), \Psi_{\delta}(u)$ satisfies the following differential equation

$$
c \Psi_{\delta}^{\prime \prime \prime}(u)+(\lambda+\delta-3 c \beta) \Psi_{\delta}^{\prime \prime}(u)+2 \beta^{2} \delta \Psi_{\delta}(u)
$$$$
-\beta(2 \lambda+3 \delta-2 c \beta-\lambda \theta) \Psi_{\delta}^{\prime}(u)=0 .
$$

proof. Applying the operator $\left(\frac{d}{d t}-\beta\right)\left(\frac{d}{d t}-2 \beta\right)$ to (36), we obtain (37).

Then from (37) and the boundary condition $\Psi_{\delta}(0)=1$, we have

$$
\Psi_{\delta}(u)=\sum_{k=0}^{2} D_{k} e^{r_{k} u}, \quad 0 \leq u \leq b,
$$

where $r_{0}, r_{1}, r_{2}$ are the solutions of equation (10). Substituting (38) into (36), we have

$$
\begin{aligned}
& \sum_{k=0}^{2} \frac{D_{k} r_{k}}{\beta-r_{k}} e^{r_{k} b}=0, \\
& \sum_{k=0}^{2} \frac{D_{k} r_{k}}{r_{k}-2 \beta} e^{r_{k} b}=0 .
\end{aligned}
$$

From the boundary condition $\Psi_{\delta}(0)=1$, we have

$$
\sum_{k=0}^{2} D_{k}=1
$$

Then solving the linear system composed by (39), (40) and (41), we can get $D_{0}, D_{1}, D_{2}$.

Corollary 5.2 When the gains distribution is exponential distribution with mean $1 / \beta$ and $\theta=0, \Psi_{\delta}(u)$ satisfies the following differential equation

$$
c \Psi_{\delta}^{\prime \prime}(u)+(\lambda+\delta-c \beta) \Psi_{\delta}^{\prime}(u)-\beta \delta \Psi_{\delta}(u)=0 .
$$

Proof. Setting $\theta=0$ in (36), we have

$c \Psi_{\delta}^{\prime}(u)+(\lambda+\delta) \Psi_{\delta}(u)-\lambda \Psi_{\delta}(b)[1-F(b-u)]$

$-\lambda \int_{0}^{b-u} \Psi_{\delta}(u+x) f_{X}(x) \mathrm{d} x=0$.

By applying the operator $\left(\frac{d}{d t}-\beta\right)$ to this equation and after some careful calculation, we obtain (42).

It follows from the boundary condition $\Psi_{\delta}(0)=1$ and equation (42) that

$$
\begin{array}{r}
\Psi_{\delta}(u)=\frac{\left(\beta-r_{0}\right) r_{1} e^{r_{1} b+r_{0} u}-\left(\beta-r_{1}\right) r_{0} e^{r_{0} b+r_{1} u}}{\left(\beta-r_{0}\right) r_{1} e^{r_{1} b}-\left(\beta-r_{1}\right) r_{0} e^{r_{0} b}}, \\
0 \leq u \leq b,
\end{array}
$$

where $r_{0}$ and $r_{1}$ are the solutions (18.)

Corollary 5.3 When the gains distribution is exponential distribution with mean $1 / \beta$ and $\theta=1, \Psi_{\delta}(u)$ satisfies the following differential equation

$c \Psi_{\delta}^{\prime \prime}(u)+(\lambda+\delta-2 c \beta) \Psi_{\delta}^{\prime}(u)-2 \beta \delta \Psi_{\delta}(u)=0$.

Proof. Similar to Corollary 2.3.

From the boundary condition $\Psi_{\delta}(0)=1$ and equation (43), we have

$$
\begin{gathered}
\Psi_{\delta}(u)=\frac{r_{1}\left(2 \beta-r_{0}\right) e^{r_{1} b+r_{0} u}-r_{0}\left(2 \beta-r_{1}\right) e^{r_{0} b+r_{1} u}}{r_{1}\left(2 \beta-r_{0}\right) e^{r_{1} b}-r_{0}\left(2 \beta-r_{1}\right) e^{r_{0} b}}, \\
0 \leq u \leq b,
\end{gathered}
$$

where $r_{0}$ and $r_{1}$ are the solutions (17)

\section{NUMERICAL ILLUSTRATION}

In this section, we illustrate the effect of dependence. Let $f_{X}(x)=e^{-x}$, then the covariance between $X$ and $W$ is given by $\operatorname{Cov}(X, W)=\frac{\theta}{\lambda} B^{2}(2,1)$, where $B(x, y)=\int_{0}^{1} t^{x-1}(1-t)^{y-1} \mathrm{~d} t$. This implies that $(X, W)$ is positive correlation when $\theta>0$, otherwise the verse.

Set $\lambda=2, c=1.1, \delta=0.1$. By comparing table I-V, we find that $V(b ; b)$ is a increase function about $b$; when $b$ is a determine constant $V(u ; b)$ is a increase function about $u$. The optimal dividend barrier $b^{*}$ decrease as $\theta$ increase.

Table VI - IX shows the probabilities that $U(t)$ reaches $b$ from $u(u<b)$ without ruin occurring. When $b-u$ is a determinant constant, the probabilities increase as $u$ increase. When $u$ and $b$ are constants, the first exit probabilities increase as $\theta$ decrease.

Table I

INFLUENCE OF $\theta$ ON $V(u ; b), b=1$

\begin{tabular}{|l||l||l||l|l|}
\hline $\mathrm{u}$ & $\theta=1$ & $\theta=0.5$ & $\theta=0$ & $\theta=-0.5$ \\
\hline 0.1 & 0.0707 & 0.0999 & 0.3554 & 0.7067 \\
\hline 0.5 & 0.3629 & 0.4362 & 1.4993 & 2.8484 \\
\hline 0.8 & 0.5956 & 0.6235 & 2.1336 & 3.9553 \\
\hline 1 & 0.7595 & 0.7156 & 2.4784 & 4.5489 \\
\hline
\end{tabular}

INFLUENCE OF $\theta$ ON $V(u ; b) \mathrm{b}=2$

\begin{tabular}{|l||l||l||l||l|}
\hline $\mathbf{u}$ & $\theta=1$ & $\theta=0.5$ & $\theta=0$ & $\theta=-0.5$ \\
\hline 0.1 & 0.0491 & 0.1270 & 0.5559 & 1.3081 \\
\hline 0.5 & 0.2516 & 0.5708 & 2.3453 & 5.2404 \\
\hline 0.8 & 0.4130 & 0.8466 & 3.3374 & 7.2099 \\
\hline 1 & 0.5267 & 1.0075 & 3.8768 & 8.2080 \\
\hline 1.2 & 0.6464 & 1.1516 & 4.3393 & 9.0214 \\
\hline 1.5 & 0.8392 & 1.3373 & 4.9198 & 9.9897 \\
\hline 1.8 & 1.0513 & 1.4848 & 5.3970 & 10.7573 \\
\hline 2 & 1.2054 & 1.5588 & 5.6719 & 11.2052 \\
\hline \multicolumn{5}{|c|}{ Table III } \\
\hline
\end{tabular}

INFLUENCE OF $\theta$ ON $V(u ; b), b=3.5$

\begin{tabular}{|l||l||l|l||l|}
\hline $\mathrm{I}$ & $\theta=1$ & $\theta=0.5$ & $\theta=0$ & $\theta=-0.5$ \\
\hline 0.1 & 0.0257 & 0.1290 & 0.6837 & 1.7196 \\
\hline 0.5 & 0.1318 & 0.5824 & 2.8841 & 6.8866 \\
\hline 1 & 0.2759 & 1.0393 & 4.7675 & 10.7750 \\
\hline 1.5 & 0.4395 & 1.4103 & 6.0502 & 13.0736 \\
\hline 2 & 0.6313 & 1.7217 & 6.9750 & 14.5328 \\
\hline 2.5 & 0.8615 & 1.9881 & 7.6897 & 15.5526 \\
\hline 3 & 1.1426 & 2.2106 & 8.28840 & 16.3462 \\
\hline 3.5 & 1.4902 & 2.3681 & 8.8127 & 17.0272 \\
\hline
\end{tabular}


Table IV

INFLUENCE OF $\theta$ ON $V(u ; b), b=4$

\begin{tabular}{|l||l||l|l||l|}
\hline $\mathrm{u}$ & $\theta=1$ & $\theta=0.5$ & $\theta=0$ & $\theta=-0.5$ \\
\hline 0.1 & 0.0204 & 0.1238 & 0.6817 & 1.7255 \\
\hline 0.5 & 0.1049 & 0.5591 & 2.8756 & 6.9102 \\
\hline 1 & 0.2196 & 0.9983 & 4.7534 & 10.8188 \\
\hline 1.5 & 0.3498 & 1.3564 & 6.0324 & 13.1182 \\
\hline 2 & 0.5204 & 1.6605 & 6.9545 & 14.5825 \\
\hline 2.5 & 0.6856 & 1.9285 & 7.6670 & 15.6058 \\
\hline 3 & 0.9094 & 2.1700 & 8.2596 & 16.4020 \\
\hline 4 & 1.5307 & 2.5574 & 9.2822 & 17.7170 \\
\hline
\end{tabular}

Table V

INFLUENCE OF $\theta$ ON $V(u ; b), b=4.5$

\begin{tabular}{|c||c||c||c||c|}
\hline $\mathrm{I}$ & $\theta=1$ & $\theta=0.5$ & $\theta=0$ & $\theta=-0.5$ \\
\hline 0.1 & 0.0162 & 0.1172 & 0.6682 & 1.7032 \\
\hline 0.5 & 0.0832 & 0.5926 & 2.8188 & 6.8208 \\
\hline 1 & 0.1742 & 0.9458 & 4.6595 & 10.6720 \\
\hline 1.5 & 0.2775 & 1.2856 & 5.9131 & 12.9486 \\
\hline 2 & 0.3987 & 1.5750 & 6.8170 & 14.3939 \\
\hline 2.5 & 0.5440 & 1.8317 & 7.5155 & 15.4040 \\
\hline 3 & 0.7215 & 2.0672 & 8.0963 & 16.1899 \\
\hline 4 & 1.2145 & 2.4839 & 9.0987 & 17.4879 \\
\hline 4.5 & 1.5575 & 2.6388 & 9.5734 & 18.0926 \\
\hline
\end{tabular}

Table VI First passage time $\theta=0$

\begin{tabular}{|c||c||c||c||c||c|}
\hline $\mathrm{I}$ & $\mathrm{b}=2$ & $\mathrm{~b}=3$ & $\mathrm{~b}=4$ & $\mathrm{~b}=5$ & $\mathrm{~b}=6$ \\
\hline 1 & 0.6939 & 0.6113 & 0.5808 & 0.5683 & 0.5629 \\
\hline 2 & 1 & 0.8810 & 0.8370 & 0.8190 & 0.8113 \\
\hline 3 & & 1 & 0.9501 & 0.9296 & 0.9209 \\
\hline 4 & & & 1 & 0.9785 & 0.9693 \\
\hline
\end{tabular}

Table VII: First passage time $\theta=1$

\begin{tabular}{|c||c||c||c||c||c|}
\hline $\mathrm{u}$ & $\mathrm{b}=2$ & $\mathrm{~b}=3$ & $\mathrm{~b}=4$ & $\mathrm{~b}=5$ & $\mathrm{~b}=6$ \\
\hline 1 & 0.3215 & 0.1374 & 0.0659 & 0.0336 & 0.0178 \\
\hline 2 & 1 & 0.4275 & 0.2050 & 0.1046 & 0.0554 \\
\hline 3 & & 1 & 0.4796 & 0.2446 & 0.1296 \\
\hline 4 & & & 1 & 0.5101 & 0.2703 \\
\hline
\end{tabular}

Table VIII First passage time $\theta=0.5$

\begin{tabular}{|c||c||c||c||c||c|}
\hline $\mathrm{u}$ & $\mathrm{b}=2$ & $\mathrm{~b}=3$ & $\mathrm{~b}=4$ & $\mathrm{~b}=5$ & $\mathrm{~b}=6$ \\
\hline 1 & 0.6502 & 0.4850 & 0.4310 & 0.4023 & 0.3861 \\
\hline 2 & 1 & 0.7967 & 0.7071 & 0.6579 & 0.6331 \\
\hline 3 & & 1 & 0.8844 & 0.8264 & 0.7912 \\
\hline 4 & & & 1 & 0.9307 & 0.8924 \\
\hline
\end{tabular}

Table IX First passage time $\theta=-0.5$

\begin{tabular}{|c||c||c||c||c||c|}
\hline $\mathrm{u}$ & $\mathrm{b}=2$ & $\mathrm{~b}=3$ & $\mathrm{~b}=4$ & $\mathrm{~b}=5$ & $\mathrm{~b}=6$ \\
\hline 1 & 0.7467 & 0.6935 & 0.6786 & 0.6732 & 0.6715 \\
\hline 2 & 1 & 0.9222 & 0.9021 & 0.8950 & 0.8926 \\
\hline 3 & & 1 & 0.9757 & 0.9680 & 0.9655 \\
\hline 4 & & & 1 & 0.9921 & 0.9575 \\
\hline
\end{tabular}

\section{REFERENCES}

[1] S. Asmussen, Ruin Probabilities, World Scientific, Singapore, 2000.

[2] H. U. Gerber, E. S. W. Shiu, “The joint distribution of the time of ruin, the surplus immediately before ruin, and the deficit at Ruin”, Insurance: Mathematics and Economics, 1997, 21: 129-137.

[3] H. U. Gerber, E. S. W. Shiu, "On the time value of Ruin”, North America Actuarial Journal, 1998, 2: 48-78.

[4] F. Dufresne, H. U. Gerber, “The surplus immediately before and at ruin, and the amount of the claim causing Ruin”, Insurance: mathematics and Economics, 1988, 7: 193-199.

[5] H. Albrecher, O. J. Boxma, "A ruin model with dependence between claim sizes and intervals”, Insurance: Mathematics and Economics, 2004, 35: 245-254.

[6] H. Albrecher, J. Teugels, "Exponential behavior in the presence of dependence in risk Theory”, Journal of Applied Probability, 2006, 43(1): 257-273.

[7] M. Boudreault, H. Cossette, D. Landriault, E.Marceau, "On a risk model with dependence between interclaim arrivals and claim sizes”, Scandinavian Actuarial Journal, 2006, 5: 265-285.

[8] B. DeFinetti, ” Su un'impostazione alternativa della teoria collettiva del rischio", Transactions of the XV International Congress of Actuaries, 1957, 2: 433443.

[9] D. C.M.Dickson, H. R. Waters, "Some optimal dividend Problems”, ASTIN Bulletin, 2004, 34: 4974.

[10] D. Landriault, “ Constant dividend barrier in a risk model with interclaim-dependent claim sizes", Insurance: Mathematics and Economics, 2008, 42: 31-38.

[11] X. S. Lin, G. E. Willmot, S. Drekic, "The classical risk model with a constant dividend barrier: Analysis of the Gerber-Shiu discounted penalty function”, Insurance: Mathematics and Economics, 2003, 33: 551-566.

[12] X. S. Lin, K. P. Pavlova, "The compound Poisson risk model with a threshold dividend strategy", Insurance: mathematics and Economics, 2006, 38: 57-80.

[13] H. Albrecher, A. L. Badescu, D. Landriault, "On the dual risk model with tax payments”, Insurance: Mathematics and Economics, 2008, 42: 1086-1094.

[14]B. Avanzi, H. U. Gerber, E. S. W.Shiu, "Optimal dividends in the dual model”, Insurance: Mathematics and Economics,2007, 41: 111-123.

[15]B. Avanzi, H. U. Gerber, "Optimal dividends in the dual model with diffusion”, ASTIN Bulletin, 2008, 38: 653-667.

[16] A. C. Y. Ng, “On a dual model with a dividend threshold”, Insurance: Mathematics and Economics, 2009, 44(2): 315-324.

[17] M. Song, R. Wu, X. Zhang, "Total duration of negative surplus for the dual model”, Applied 
stochastic model in business and industry 2008 24: 591-600.

[18] R. B. Nelsen, “An introduction to Copulas”, second edition, Springer-Verlag, New York, 2006.

[19] D. C. M. Dickson, C. Hipp, "Ruin probabilities for Erlang(2)risk process”, Insurance: Mathematics and Economics, 1998, 22: 251-262.

Hua Dong a Ph.D candidate from Central South University. Her research interests include risk theory and mathematical finance.
Zaiming Liu received Bachelor of Science from Hunan Normal University, China in 1981, Master of Science and Ph.D in probability and statistics from Changsha Tiedao College(Central South University now), China in 1984 and 1988, respectively. His research interests include Markov process and its application, risk theory, and queueing theory.

Currently, he is the dean of school of mathematical sciences and computing technology, Central South University, P.R. China. He is surpported by National Science Foundation of China (No.10971230) and the National Science Foundation of Hunan (No.08JJ3004). 